BULLETIN OF THE

AMERICAN MATHEMATICAL SOCIETY

Volume 80, Number 1, January 1974

\title{
BEST APPROXIMATION BY COMPACT OPERATORS. II
}

\author{
BY RICHARD HOLMES, BRUCE SCRANTON AND JOSEPH WARD
}

Communicated by Robert Bartle, July 23, 1973

Introduction. Let $H$ be an infinite-dimensional complex Hilbert space, and let $\mathscr{B}(H)$ (resp. $\mathscr{C}(H))$ be the algebra of all bounded (resp. compact) linear operators on $H$. It is well known [4], [6] that $\mathscr{C}(H)$ is proximinal in $\mathscr{B}(H)$, that is, every $T \in \mathscr{B}(H)$ has a best approximation from the subspace $\mathscr{C}(H)$. Indeed, it has recently been noted by Fakhoury [2] that there is a continuous selection for the associated metric projector. We denote the metric complement of $\mathscr{C}(H)$ in $\mathscr{B}(H)$ by $\mathscr{C}(H)^{0}$; by definition, $\mathscr{C}(H)^{0}=\{T \in \mathscr{B}(H):\|T\|=\operatorname{dist}(T, \mathscr{C}(H))\} \equiv\{T \in \mathscr{B}(H): 0$ is a best compact approximation to $T\}$. Such operators (other than 0 ) are at maximum possible distance from $\mathscr{C}(H)$; they may thus be called "anti-compact" (called extremally noncompact by Coburn [1]). Every operator $T$ is the sum of a compact operator and an anti-compact operator; we can give one such splitting explicitly in terms of the polar decomposition of $T$. It is the purpose of this note to announce a series of results on the contents and structure of $\mathscr{C}(H)^{0}$, with particular reference to the proper subset consisting of those operators for which 0 is the unique best compact approximation that commutes with the given operator. Full details and proofs of the theorems will appear elsewhere.

1. Failure of unicity. The seminal result that motivates and directs our subsequent investigation is the rather striking failure, in all cases, of uniqueness of best compact approximations.

THEOREM 1. Let $T$ be any noncompact operator in $\mathscr{B}(H)$. Then the set of all best compact approximations to $T$ is a (closed, bounded) convex set of infinite dimension.

It is clear that the metric complement of a Chebyshev subspace of a Banach space $X$ is a (closed) nowhere dense subset of $X$, and simple examples show that the metric complement of a non-Chebyshev subspace

AMS (MOS) subject classifications (1970). Primary 41A65, 47B05, 47B20; Secondary 47A30, 47D20.

Key words and phrases. Best approximation, compact operator, anti-compact operator, metric complement of the compact operators, commuting best compact approximation.

Copyright $@$ American Mathematical Society 1974 
may well have nonempty interior. Thus the next result is unexpected (however, see Theorem 7 below):

THEOREM 2. $\mathscr{C}(H)^{0}$ is nowhere dense in $\mathscr{B}(H)$.

COROLlaRY 1. The set of operators in $\mathscr{B}(H)$ which fail to attain their norm on the unit ball of $H$ is nowhere dense in $\mathscr{B}(H)$.

The corollary derives its interest by contrast with a result of Lindenstrauss [5], which asserts that the set of operators which do attain their norm is dense in $\mathscr{B}(H)$ (this latter fact being valid if $H$ is replaced by an arbitrary reflexive Banach space). Now from the corollary we see that the set of operators attaining their norm is actually of second category in $\mathscr{B}(H)$, and thus we obtain an independent proof of a slightly stronger form of Lindenstrauss' result in the Hilbert space case.

2. Distance formulas. In [3], [4] formulas were given for $\operatorname{dist}(T, \mathscr{C}(H))$, for any $T \in \mathscr{B}(H)$. In particular, Gohberg and KreIn showed that $\operatorname{dist}(T, \mathscr{C}(H))$ is the essential spectral radius of the positive part of $T$. We now consider a class of operators for which this result can be improved. Let $r_{e}(T)$ be the essential spectral radius of $T \in \mathscr{B}(H)$, and let us say that $T$ is essentially normaloid if $r_{e}(T)=\|T\|$.

THEOREM 3. For every $T \in \mathscr{B}(H)$, we have $r_{e}(T) \leqq \operatorname{dist}(T, \mathscr{C}(H))$. The class $\mathscr{D}$ of operators $T$ for which equality holds here is a closed, balanced, nowhere dense cone in $\mathscr{B}(H) . \mathscr{D}$ consists of those operators having an essentially normaloid compact perturbation, and in particular, $\mathscr{D}$ contains every seminormal operator.

Since this result is established independently of [3], and since $\operatorname{dist}(T, \mathscr{C}(H))=\operatorname{dist}\left(\left(T^{*} T\right)^{1 / 2}, \mathscr{C}(H)\right)$ always holds, it follows that Theorem 3 includes the Gohberg-Krein formula, and hence Theorem 5.1 of [1]. That $\mathscr{D}$ contains every seminormal operator is an easy consequence of the work of Salinas [7]. On the other hand, $\mathscr{D}$ does not contain all weighted shifts.

THEOREM 4. Let $T$ be a (unilateral or bilateral) weighted shift with weights $a_{n}>0$. Then

$$
\operatorname{dist}(T, \mathscr{C}(H))=\limsup _{|n| \rightarrow \infty} a_{n} .
$$

3. Anti-compact operators. What kinds of operators in $\mathscr{B}(H)$ are anti-compact? From [4] it follows, for example, that all partial isometries of infinite rank are anti-compact. We also have

THEOREM 5. Every essentially normaloid operator is anti-compact. 
These two types of operators do not yet exhaust the metric complement $\mathscr{C}(H)^{0}$. For example, certain quasi-nilpotent weighted shifts are anticompact.

THEOREM 6. The metric complement $\mathscr{C}(H)^{0}$ contains a homeomorph of the Calkin algebra.

It is an open question whether $\mathscr{C}(H)^{0}$ is actually homeomorphic to a Banach space.

TheOREM 7. $\mathscr{C}(H)^{0}$ is strong operator dense in $\mathscr{B}(H)$.

4. Uniqueness of commuting best approximations. The proof of Theorem 3 shows that every seminormal operator $T$ has a best compact approximation that commutes with $T$. Motivated by this observation and by Theorem 1 we ask which operators have unique commuting best compact approximations. Now in general, on account of Lomonosov's theorem [6], this is not a trivial problem. However, anti-compact operators have, by definition, a commuting best compact approximation, namely 0 . To study this situation in more detail, we introduce two classes of operators in $\mathscr{B}(H): Z U C=\{T \in \mathscr{B}(H): 0$ is the unique compact operator that commutes with $T\}$ and $Z U C A=\{T \in \mathscr{B}(H): 0$ is the unique commuting best compact approximation to $T$ \}. Clearly,

$$
Z U C \cap \mathscr{C}(H)^{0} \subset Z U C A \subset \mathscr{C}(H)^{0},
$$

and these inclusions are proper.

Below we identify several interesting classes of $Z U C A$ operators. These operators are all subnormal and eigenvalueless, hence are at least anti-compact by Theorem 5. Further examples of $Z U C A$ operators are obtained as the adjoints of these operators, and hence may be only seminormal and have nonempty point spectrum. These examples should be contrasted with

THEOREM 8. No hyponormal operator with nonempty point spectrum can have a unique commuting best compact approximation.

THEOREM 9. Each of the following classes of operators belongs to $Z U C \cap \mathscr{C}(H)^{0}$ :

(a) normal and eigenvalueless;

(b) isometric and eigenvalueless;

(c) analytic Toeplitz operators, defined by a schlicht function;

(d) multiplications by bounded schlicht functions on some Bergman space;

(e) the discrete Cesàro operator.

The following general principle is useful for some of these cases. 
Lemma. Let $T$ be an eigenvalueless operator in $\mathscr{B}(H)$ such that $T^{*}$ has so many simple eigenvalues that the corresponding eigenvectors are fundamental in $H$. Then $T$ belongs to $Z U C$ (although not necessarily to $\left.\mathscr{C}(H)^{0}\right)$.

Note that, although the result of Shields and Wallen [8, Theorem 2] implies that their multiplication operators $M_{z}$ belong to $Z U C$, the preceding lemma is applicable to a more general situation where their condition (c) is significantly weakened and condition (d) is eliminated.

Unfortunately, it is not the case that every eigenvalueless subnormal operator belongs to $Z U C A$. In fact, we have

EXAMPLE. There exists an eigenvalueless quasi-normal operator not in the class $Z U C A$.

To see that the class $Z U C A$ properly contains the class $Z U C \cap \mathscr{C}(H)^{0}$, let $U$ be the ordinary unilateral shift, and consider the operator $2 U \oplus U$. More complicated examples exist: these are certain eigenvalueless weighted shifts that fail to attain their norm (compare with Corollary 3 below). In general, any weighted shift in either $Z U C$ or $Z U C A$ must be eigenvalueless.

THEOREM 10. A shift with weights $a_{n}>0$ fails to belong to ZUC exactly when there exists some $k \geqq 1$ such that

$$
\lim _{n \rightarrow \infty}\left(a_{n+1} a_{n+2} \cdots a_{n+k}\right)=0 .
$$

COROLlaRY 2. Every hyponormal weighted shift belongs to ZUCก $\mathscr{C}(H)^{0}$.

Also, as a particular consequence of a (rather complicated) sufficient condition for a weighted shift to belong to $Z U C A$, we have

COROLlaRY 3. Every anti-compact weighted shift that attains its norm belongs to $Z U C A$.

The relationship between the metric complement $\mathscr{C}(H)^{0}$ and its subset $Z U C A$ is interesting. For example, if a nonzero operator belongs to $Z U C A$, it must not admit any compact direct summand; no stronger necessary condition is known. In a different vein, the possibility that $Z U C A$ is dense in $\mathscr{C}(H)^{0}$ is an intriguing but open question. Certainly $Z U C A$ is not closed. There is, for example, a sequence of eigenvalueless normal operators that converges to the identity operator. Such a phenomenon exhibits the delicate and discontinuous nature of the $Z U C A$ property: we have a sequence of operators, each of whose set of commuting best compact approximations is zero-dimensional, but whose (norm) limit has an infinite-dimensional set of commuting best compact approximations. 


\section{REFERENCES}

1. L. Coburn, Weyl's theorem for non-normal operators, Michigan Math. J. 13 (1966), 285-288. MR 34 \#1846.

2. H. Fakhoury, Projections de meilleure approximation continues dans certains espaces de Banach, C.R. Acad. Sci. Paris 276 (1973), A45-A48.

3. I. Gohberg and M. Krein, Introduction to the theory of linear nonselfadjoint operators in Hilbert space, "Nauka", Moscow, 1965; English transl., Transl. Math. Monographs, vol. 18, Amer. Math. Soc., Providence, R.I., 1969. MR 36 \#3137; 39 \#7447.

4. R. Holmes and B. Kripke, Best approximation by compact operators, Indiana Univ. Math. J. 21 (1971/72), 255-263. MR 45 \#5718.

5. J. Lindenstrauss, On operators which attain their norm, Israel J. Math. 1 (1963), 139-148. MR 28 \#3308.

6. C. Pearcy and A. Shields, Compact operators have hyperinvariant subspaces (to appear).

7. N. Salinas, Operators with essentially disconnected spectrum, Acta Sci. Math. (Szeged) 33 (1972), 193-205.

8. A. Shields and L. Wallen, The commutants of certain Hilbert space operators, Indiana Univ. Math. J. 20 (1970/71), 777-788. MR 44 \#4558.

Department of Mathematics, Purdue University, Lafayette, Indiana 47907 